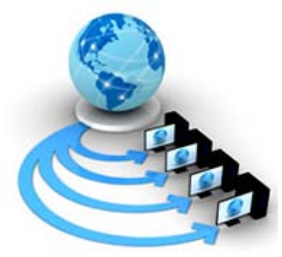

Volume 8, No. 7, July - August 2017

International Journal of Advanced Research in Computer Science

REVIEW ARTICLE

\author{
Available Online at www.ijarcs.info
}

\title{
RECOMMENDATION SYSTEMS: A REVIEW REPORT
}

\author{
Kunika Arora \\ (M.Tech student) \\ Department of CSE, Panipat Institiute of Engineering \& \\ Technology, Samalkha, Haryana, India
}

\author{
Shekhar Singh \\ (Assistant Professor) \\ Department of CSE, Panipat Institiute of Engineering \& \\ Technology, Samalkha, Haryana, India
}

\author{
Dr. Vikram Bali \\ (Associate Professor) \\ Department of CSE, Panipat Institiute of Engineering \& \\ Technology, Samalkha, Haryana, India
}

\begin{abstract}
To deal with information overloaded problem on the internet, there is need to filter, efficient and accurately deliver the pertinent information. So, recommendation system is used to resolve this problem. Recommendation system filters out the information fragment according to user behaviour or interest. Recommendation system can predict the interest of the user and also predict that the user would prefer any particular item or not. For both users and service providers, recommendation system is profitable and it is also effective in increasing sales of many products. This paper explores many recommendation techniques and compares their characteristics, strength and weaknesses to enhance the execution of the recommendation system.
\end{abstract}

Keywords: singular value decomposition (SVD), content-based (CB)

\section{INTRODUCTION}

Because of large number of visitors and huge amount of digital information available on internet, data overloaded problem is created and it make difficult for someone to do something of his interest at the internet. There are some information retrieval systems are also available like Google, Alta-Vista etc. but in these systems prioritization and individualization are not present. So we are in need for the recommendation systems to resolve all these kind of problems. To manage the issue of data over-burden [1], recommendation system filters out the information fragment according to user behavior or interest [2]. Based on user profile, recommendation system can predict the interest of the user and also predict that the user would prefer any particular item or not.

To find and select the product or item in any online shopping application, recommendation system reduces the transaction cost [3]. For both users and service providers, recommendation system is profitable [4] and it is also effective in increasing sales of many products. In improving the decision making process and efficient quality also recommendation system have been proved [5]. Recommendation system is beneficial in scientific laboratory, by allowing the user to move past index seeks. So, we require proficient and precise recommendation techniques within a system.

\section{RELATED WORK}

During information overloaded situation, recommendation system is characterized as a Decision making procedure for clients. A different characteristic of different prediction techniques is also explores. Recommendation system searching large volume of information to provide users with personalized content [6]. It enables the client to look the thing of client to intrigue and preference. Recommendation system guide consumers through locating products users will like by using hand-coded knowledge or minded knowledge learned from the behavior of consumers [7]. When user have no personal knowledge or choices then recommendation system use other user's recommendation to make choices for that particular user [8]. It handles the information overloaded problems of the user by providing them exclusive content and services recommendation. Collaborative filtering, content-based filtering and hybrid filtering are the approaches to develop the recommendation system [9-10]. Among all these filtering approaches, collaborative filtering is mostly used and preferred for recommendation system. It is the mature and commonly implemented for recommendation system. In this filtering, the other user which have the similar taste of item with the active user, collaborative filtering recommends that item to the particular user. This filtering have been implemented in different application areas like Group Lens, Ringo etc. group lens employ the collaborative methods to locate articles from news database [11]. Ringo is a music application. To build the user profile, Ringo also uses the collaborative filtering. It depends on user rating on the music albums [12]. To improve its recommendation, Amazon uses topic diversification algorithm. We used the intra-list similarity metric to assess the topical diversity of recommendation lists and the topic diversification approach for decreasing the intra-list similarity [13]. To reduce the scalability problems, Amazon uses the collaborative filtering. Depending on the purchasing background of the user, it prescribes alternate things to the client. By using 
product-to-produce matrix, it generates a table of similar products.

In content-based filtering, upon user's information, it bases the prediction and matches the content resources to user characteristics. Like collaborative filtering, it does not depend on predictions of other user with same items. It only depends on that particular or active user information [14]. Collaborative filtering (CF) is a technique commonly used to build personalized recommendations on the Web [15]. Some popular websites that make use of the collaborative filtering technology include Amazon, Netflix, iTunes, IMDB. Fab uses the content-based filtering and depends on different user's rating to make a preparation set. Letizia is another example which based on the content-based filtering and helps the users to find data on the internet [16]. By tracking the browsing pattern of the user, it predicts the pages of the user interest. By using naive Bayesian classifier, Pazzani [17] designed an agent that predicts the user's interest web pages. By rating different pages, agent allows a user to provide training instances. A neural network is also described in use net news applications to predict the interest of the user [18].

These two filtering techniques have so many advantages but there are some limitations also identified in these techniques. The restrictions of the content-based filtering techniques are- (i) limited content analysis, (ii) sparsity of data (iii) overspecialization. The limitations of the collaborated filtering techniques are (i) sparsity problems (ii) cold start etc.

Because of all these problems the quality of recommendation is get decreased. With a specific end goal to enhance the exactness and execution of the recommendation system, two or more filtering techniques are combined to overcome all these problems. This is called hybrid filtering. In this two or more filtering techniques are combined to combine their strength and filtering out their weaknesses. This approach can be classified as (i) mixed hybrid (ii) weighted hybrid (iii) switching hybrid (iv) Cascaded hybrid (v) feature-combination hybrid and (vi) meta-level hybrid.

In order to increase the quality of recommendations, user navigation patterns are used to capture similar behaviors of users $[19,20]$. These techniques integrate two or more filter outing techniques so that it will harness their strengths at the same time as levelling out their corresponding weaknesses. Collaborative filtering is the most widely and successfully used filtering technique and it has cross- genre ability or outside the box recommendation ability that is independent of any machine-readable representation of the items being recommended. [21].

Nowadays these filtering are implemented differently. Their outputs are combined based on the predictions of both or the attributes of collaborative filtering are adding to contentbased filtering and vice-versa. Finally by combining the both properties, a new model is generated .As we already studied about the issue of icy begin and sparsity of information in filtering techniques. These problems are reduces in cascaded hybrid recommendation techniques by including the appraisals, components and data about the items [22]. Different characteristics and potentials of different prediction techniques in recommendation systems is explore in order to serve as a compass for research and practice in the field of recommendation systems. [23] In
Ziegler [24], a hybrid recommendation technique is planned to tackle the data sparsity problem and to find prediction of similar users, content based filtering is also used. A collaborative filtering is combined with an information filtering agent in [25] and to integrate the content based filtering agent and collaborated filtering, a framework is also proposed. To remove the new user problems of content based filtering and average user collaborated filtering approaches, a hybrid recommendation algorithm is proposed by many applications [26]. In [27], a music recommendation system is used which proposed the tagging information, play counts and social relation. In [28] lee embedded social information into collaborative filtering to determine the number of neighbours that can be automatically connected on a social platform. In a unified framework, item rating and features are proposed by Condiff [29].

\section{PHASES OF RECOMMENDATION PROCESS}

\section{A. Information collection phase}

In this phase, the profile of the user is generated to collect the data related to the user. This work derived from user's behavior and the data that is access by the user. After constructing the profile of the user accurately, this recommendation agent gets start functioning. To provide the best recommendation for the user, it s required to get the right data related to the user in their profile. There are 3 types of feedback in the system. These are (i) explicit (ii) implicit and (iii) hybrid feedback. By combining both feedbacks, hybrid feedback is obtained. The summary of the user is the collection of the data of the user like their skills, abilities, interest, learning styles etc. if the system has accurate information of user's interest then a recommendation system will give a accurate recommendations to the user.

\section{- $\quad$ Explicit feedback}

More the quantity of the items more the accuracy of the recommendation system received. The only drawback of this method is that is required more efforts from the user. But still this feedback gives more reliable information and higher quality, accuracy of the recommendation.

\section{- Implicit feedback:}

In this, more efforts from the user are not required. By monitoring the user's behavior, data access, browser history, time spent on some web pages, links, content of e-mail and click button among others etc, the system automatically predict the user preferences. This method does not provide reliable and accurate information like explicit feedback.

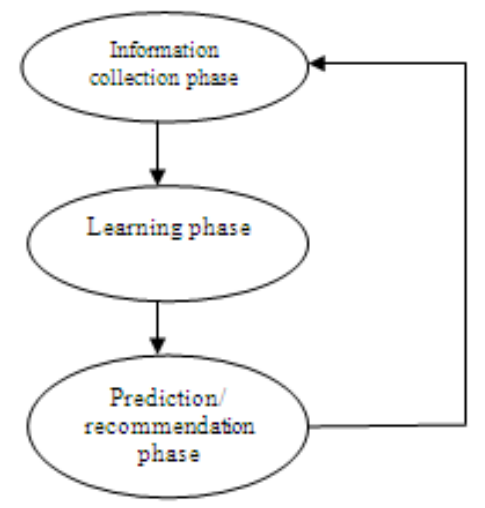

Figure 1 Recommendation phases 


\section{- Hybrid feedback}

By combining both understood and unequivocal feedbacks we get hybrid feedback. In this, the strength of both feedbacks are combined and weaknesses of the feedbacks are minimized so that to get the better performance of the system.

\section{- Learning phase}

A learning algorithm is utilized to the filter and from the feedback collected in information collection phase, user's features are exploited.

\section{- Prediction/recommendation phase}

It utilizes a learning to channel and adventure the client's elements from the criticism accumulated in data gathering stage..

\section{RECOMMENDATION FILTERING TECHNIQUES}

For useful and good recommendation system for the users, the utilisation of accurate \& effective recommendation techniques is quite necessary for the system. Figure 2 shows the different-different recommendation filtering techniques.

\section{A. Content-based filtering}

In content-based filtering, upon user's information, it bases the prediction and matches the content resources to user characteristics. Like collaborative filtering, it does not depend on predictions of other user with same items. It only depends on that particular or active user information. Fab uses the content-based filtering and depends on different user's rating to make a preparation set. Letizia is another example which depending on the content-based filtering and helps the users to find data on the internet. By tracking the browsing pattern of the user, it predicts the pages of the user interest. When we want to recommended the web pages, news then content based filtering is best suited. In this, recommendation is based on user profile which contains features of the items, user history etc. it uses vector space model and probability model, decision tree and neural networks. In Content based filtering, for recommendation it does not need the other user's profile. An item that we search online, its features need to have a depth knowledge and information in the profile.

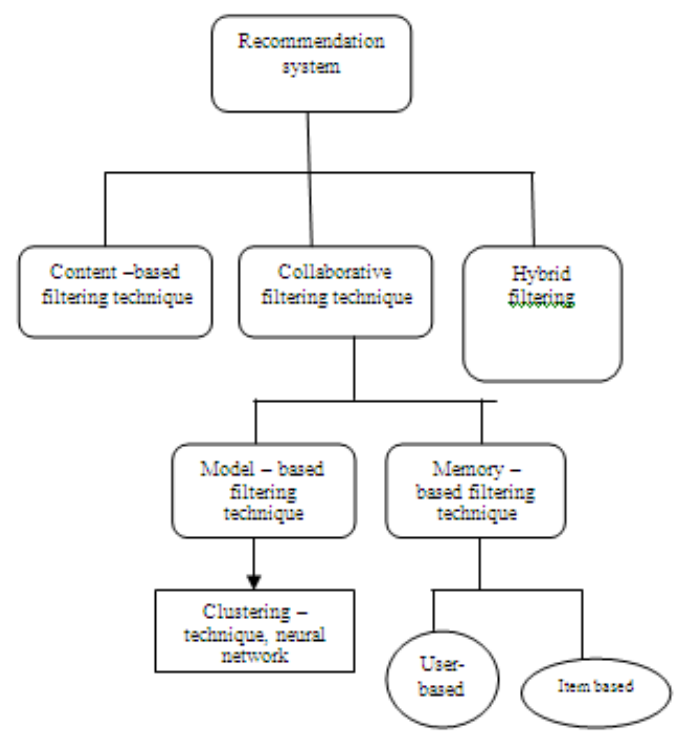

Figure 2 Recommendation techniques
- Pros and Cons of content-based filtering techniques:

If users do not rate any item even then content based filtering has the ability to provide new recommendation to them. CB filtering is used to decrease most of the drawbacks or challenges of collaborative filtering. If user preference changes or not found in database even then the accuracy of the system is not affected. In this, the profile of the user need to be organized very well and description of the items is also required. More the data is descriptive more the system is effective. Content over-specialization is the major issue of content based filtering. In this, if users defined any item in their profiles then he is restricted to getting recommendation similar to that item.

\section{B. Collaborative filtering}

Collaborative filtering is mostly used and preferred for recommendation system. It is the mature and commonly implemented for recommendation system. In this filtering, the other user which have the similar taste of item with the active user, collaborative filtering recommends that item to the particular user. This filtering has been implemented in different application areas like Group Lens, Ringo etc. Group lens employ the collaborative methods to locate articles from news database [30]. Ringo is a music application. To build the user profile, Ringo also uses the collaborative filtering. It depends on user rating on the music albums. To improve its recommendation, Amazon uses topic diversification algorithm. To reduce the scalability problems, Amazon uses the collaborative filtering. Depending on the purchasing background of the user, it prescribes alternate things to the client. By using product-to-produce matrix, it generates a table of similar products. In this, users get recommendation only for those items that are not rated before but positively rated by other user. Recommendation produced by this technique can be either prediction or recommendation. Two catagories under which collaborative filtering is classified are: memory based and model based.

\section{- Model-based techniques:}

These techniques are used for building this model process. For effective performance of the filtering is achieved by the previous rating of the item. In this, the recommendation output of the user is similar to neighbor recommendation techniques. Examples of this technique are singular value decomposition (SVD), matrix completion technique, regression and clustering. Sparsity problem in recommendation systems is also removed in this technique.

\section{- Memory based techniques}

In this technique, recommendation is based on already rated items by neighbors of the user. By combining the preferences of diff-diff users, recommendations are generated. This technique is so effective and reliable. So, this is used in real life applications. The two ways to attain memory based CF through user based and item based techniques. When two users give the rating to a particular product, then based on their rating of similarity it compute the predicted rating of the item in the user based technique. In item based technique, there is prediction is done by comparing the similarity between items. Similarity between users is not into consideration. 


\section{(i) Problems of collaborative filtering techniques}

There are so many advantages and drawbacks of the Collaborative filtering. Advantage is that it can perform in areas where computer system cannot analyze. Problems associated with this are discussed below:

\section{- Cold-start problem}

To make predictions for the users, when recommender does not have enough information about users or items then this type of problem is produced. Because of this major drawback, the performance of the recommendation system is reduced. If new user does not rate any item or product, the profile of that user will assume to be empty.

\section{- Data sparsity problem}

In database, when a few number of item are rated from the total number of items and recommender has less amount of information about the user or the item, then this type of problem is occur. Because of this problem, a weak recommendation is generated. This also increase the coverage problem in the system.

\section{- Synonymy}

Because of the similar type of items and names in the database, this problem is occurs. Sometimes it is difficult to find the difference between two items like men cloth and men wear. Both items are closely related to each other. So there is a difficulty to differentiate between similar types of products. Different methods such as singular value decomposition (SVD), automatic term expansion are used to solve this problem. Sometimes because of this problem, the performance of the recommendation system is degraded also.

\section{(ii) Examples of collaborative systems}

- Ringo is a music application. To build the user profile, Ringo also uses the collaborative filtering. It depends on user rating on the music albums .A list of 125 artists is given to the user, when user entered the system to rate them according to his choice and how much he like to listen them. There is two sessions in ringo. In first session, highly rated artists are rated by the user. In second session, a random selection of items is generated so that all artists are rated at some point.

- Group Lens employs the collaborative methods to locate articles from news database .It recommends the use-net news. Netnews has a short lifetime. This is the main challenge addressed by the system. Netnews are based on News exist in the system.

- Amazon.com To improve its recommendation, Amazon uses topic diversification algorithm. To reduce the scalability problems, Amazon uses the collaborative filtering. Depending on the purchasing background of the user, it prescribes alternate things to the client. By using product-to-produce matrix, it generates a table of similar products. It is an ecommerce recommendation technique. It enhances the execution of the recommendation by gathering the data about the user by checking his browsing history, time spent on any web etc.

\section{Hybrid filtering}

To overcome the limitations of the recommendation system, a hybrid filtering technique is used by combining different types of filtering techniques and improving system performance. When we combine two or more algorithms, provide effective and accurate recommendation than a single algorithm. It also overcomes the disabilities of the single algorithm by combining more algorithms. This approach can be classified as (i) mixed hybrid (ii) weighted hybrid (iii) switching hybrid (iv) Cascaded hybrid (v) featurecombination hybrid and (vi) meta-level hybrid. Some of them are explained below:

\section{- Weighted hybridization}

To generate a recommendation output, in weighted hybridization combines the results of many recommendations. P-tango is the example of this hybridization. In this, content based and collaborated recommender is used. Firstly they generate same weight and then adjust the weight according to predictions. Strength of all recommender systems are combine in this technique in a straight forward way.

\section{- Switching hybridization:}

This technique provides good rating to the recommendation system. This approach is delicate to quality and shortcoming of the recommender. Because of switching criterion, it introduces more complexity to the recommendation system. Daily-Learner is the example of this technique.

\section{- Cascade hybridization:}

In this, the recommendation of one technique is refined by another recommendation technique. The result of one system is provided or refined to another system by next recommendation technique. This technique overcomes the noise present in the system and provides efficient results of the recommendation system. The example of this technique is EntreeC that used a collaborated recommender.

\section{CONCLUSION}

Information overloaded is a serious problem in information retrieval systems. Recommender system resolve the problem of information overloaded and open new opportunities of retrieving individualized information on the internet and access the products and services to the users. In this paper, described content-based and collaborative filtering and to enhance the execution of the system, compared their weaknesses and strengths with hybrid filtering. We also discussed various algorithms to generate recommendation and measuring the quality, performance of the recommendation system.

\section{REFERENCES}

[1] Konstan JA, Riedl J. Recommender systems: from algorithms to user experience. User Model User-Adapt Interact 2012;22:101-23.

[2] Pan C, Li W. Research paper recommendation with topic analysis. In Computer Design and Applications IEEE 2010;4, pp. V4-264.

[3] Pu P, Chen L, Hu R. A user-centric evaluation framework for recommender systems. In: Proceedings of the fifth ACM confer-ence on Recommender Systems (RecSys'11), ACM, New York, NY, USA; 2011. p. 57-164.

[4] $\mathrm{Hu}$ R, Pu P. Potential acceptance issues of personality-ASED recommender systems. In: Proceedings of ACM conference on recommender systems (RecSys'09), New York City, NY, USA; October 2009. p. 22-5.

[5] Pathak B, Garfinkel R, Gopal R, Venkatesan R, Yin F. Empirical analysis of the impact of recommender systems on sales. J Manage Inform Syst 2010;27(2):159-88.

[6] Rashid AM, Albert I, Cosley D, Lam SK, McNee SM, 
Konstan JA et al. Getting to know you: learning new user preferences in recommender systems. In: Proceedings of the international conference on intelligent user interfaces; 2002. p. 127-34.

[7] Schafer JB, Konstan J, Riedl J. Recommender system in ecommerce. In: Proceedings of the 1st ACM conference on electronic commerce; 1999. p. 158-66.

[8] Resnick P, Varian HR. Recommender system's. Commun ACM 1997;40(3):56-8. http://dx.doi.org/10.1145/245108.24512.

[9] Acilar AM, Arslan A. A collaborative filtering method based on Artificial Immune Network. Exp Syst Appl 2009;36(4):8324-32.

[10] Chen LS, Hsu FH, Chen MC, Hsu YC. Developing recommender systems with the consideration of product profitability for sellers. Int J Inform Sci 2008;178(4):103248.

[11] Jalali M, Mustapha N, Sulaiman M, Mamay A. WEBPUM: a web-based recommendation system to predict user future move- ment. Exp Syst Applicat 2010;37(9):6201-12.

[12] Adomavicius G, Tuzhilin A. Toward the next generation of recommender system. A survey of the state-of-the-art and possible extensions. IEEE Trans Knowl Data Eng 2005;17(6):734-49.

[13]Ziegler CN, McNee SM, Konstan JA, Lausen G. Improving recommendation lists through topic diversification. In: Proceedings of the 14th international conference on World Wide Web; 2005. p. 22-32.

[14] Min SH, Han I. Detection of the customer time-variant pattern for improving recommender system. Exp Syst Applicat 2010;37(4):2911-22.

[15] Celma O, Serra X. FOAFing the Music: bridging the semantic gap in music recommendation. Web Semant: Sci Serv Agents World Wide Web 2008;16(4):250-6.

[16]Lieberman H. Letizia: an agent that assists web browsing. In: Proceedings of the 1995 international joint conference on artificial intelligence. Montreal, Canada; 1995. p. 924-9.

[17] Pazzani MJ. A framework for collaborative, content-based and demographic filtering. Artific Intell Rev 1999;13:393408, No. 5(6).

[18] Jennings A, Higuchi H. A personal news service based on a user model neural network. IEICE Trans Inform Syst 1992;E75- D(2):198-209.

[19] Murat G, Sule GO. Combination of web page recommender systems. Exp Syst Applicat 2010;37(4):2911-22.

[20] Mobasher B. Recommender systems. Kunstliche Intelligenz.
Special Issue on Web Mining, BottcherIT Verlag, Bremen, Germany, vol. 3; 2007. p. 41-3.

[21] Al-Shamri MY, Bharadwaj KK, "Fuzzy-genetic approach to recommender systems based on a novel hybrid user model. Expert Syst Appl 2008;35(3):1386-99.

[22] Mican D, Tomai N. Association ruled-based recommender system for personalization in adaptive web-based applications. In: Proceedings of the 10th international conference on current trends in web engineering (ICWE'10), Berlin, SpringerVerlag, 2010; p. 85-90.

[23] Ghazantar MA, Pragel-Benett A. A scalable accurate hybrid recommender system. In: the 3rd International conference on knowledge discovery and data mining (WKDD 2010), IEEE Computer Society, Washington, DC, USA. < http://eprint.ecs.so- ton.ac.uk/18430>.

[24]Ziegler CN, Lausen G, Schmidt-Thieme L. Taxonomy-driven computation of product recommendations. In: Proceedings of the 13th international conference on information and knowledge management (CIKM '04), Washington, DC, USA; 2004. p. 406- 15.

[25] Sarwar BM, Konstan JA, Herlocker JL, Miller B, Riedl JT. Using filtering agents to improve prediction quality in the grouplens research, collaborative filtering system. In: Proceedings of the ACM conference on computer supported cooperative work. NY (USA): ACM New York; 1998. p. 345-54.

[26] Burke R. Hybrid web recommender systems. In: Brusilovsky P, Kobsa A, Nejdl W, editors. The adaptive web, LNCS 4321. Berlin Heidelberg, Germany: Springer; 2007. p. 377-408. http:// dx.doi.org/10.1007/978-3-540-72079-9_12.

[27]Cunningham P, Bergmann R, Schmitt S, Traphoner R, Breen S, Smyth B. "WebSell: Intelligent sales assistants for the World Wide Web. In: Proceedings CBR in ECommerce, Vancouver BC; 2001. p. 104-9.

[28] Konstan I, Stathopoulos V, Jose JM. On social networks and collaborative recommendation. In: The proceedings of the 32nd international ACM conference (SIGIR'09), ACM. New York, NY, USA; 2009. p.195-202.

[29]Lee DH, Brusilovsky P. Social networks and interest similarity: the case of CiteULike. In: Proceedings of the 21st ACM conference on Hypertext and Hypermedia (HT'10). ACM. New York, NY, USA; 2010. p. 151-6.

[30] Condiff MK, Lewis DD, Madigan D, Posse C. Bayesian mixed-effects models for recommender systems. In: Proceedings of ACM SIGIR workshop of recommender systems: algorithm and eval-uation; 1999. 